\title{
Rapid and Facile Quantitation of Polyplex Endocytic Trafficking
}

\author{
Mihael Lazebnik ${ }^{\mathrm{a}, \dagger}$ and Daniel W. Pack ${ }^{b, c, *}$
}




\section{ABSTRACT}

Design of safe and effective synthetic nucleic acid delivery vectors such as polycation/DNA or polycation/siRNA complexes (polyplexes) will be facilitated by quantitative understanding of the mechanisms by which such materials escort cargo from the cell surface to the nucleus. In particular, the mechanisms of cellular internalization by various endocytosis pathways and subsequent endocytic vesicle trafficking have been shown to strongly affect nucleic acid delivery efficiency. Fluorescence microscopy and subcellular fractionation methods are commonly employed to follow intracellular trafficking of biomolecules and nanoparticulate delivery systems such as polyplexes. However, it is difficult to obtain quantitative data from microscopy and subcellular fractionation is experimentally difficult and low throughput. We have developed a method for quantifying the transport of polyplexes through important endocytic vesicles. The method is based on polymerization of 3,3'-diaminobenzidine by endocytosed horseradish peroxidase, causing an increase in the vesicle density, resistance to being solubilized by detergent and quenching of fluorophores withing the vesicles, which makes them easy to separate and quantify. Using this method in HeLa cells, we have observed polyethylenimine/siRNA polyplexes initially appearing in early endosomes and rapidly moving to other compartments within 30 min post-transfection. At the same time, we observed the kinetics of accumulation of the polyplexes in lysosomes at a similar rate. The results from the new method are consistent with similar measurements by confocal fluorescence microscopy and subcellular fractionation of endocytic vesicles on a Percoll gradient. The relative ease of this new method will aid investigation of gene delivery mechanisms by providing the means to rapidly quantify endocytic trafficking of polyplexes and other vectors. 
Keywords: gene delivery; intracellular trafficking; polyplexes

\section{INTRODUCTION}

Human gene therapy holds tremendous therapeutic potential. Yet clinical implementation of this revolutionary technology is hindered by the need for safe and efficient methods for delivery of nucleic acids. Synthetic gene delivery vectors avoid many of the limitations of the commonly employed recombinant viral vectors. In particular, polymer-based vectors can be noncytotoxic and non-immunogenic, carry large gene sequences, and are more amenable to largescale production. Current polymers, however, do not exhibit the gene delivery efficiency required for clinical translation. Design of more efficient materials, therefore, is necessary and requires understanding of the mechanisms by which polymers carry genetic material through a target cell and deliver it efficiently to the nucleus.

Polyethylenimine (PEI) has been studied as a gene delivery vector since 1995 [1,2] and provides high gene delivery efficiency compared to other off-the-shelf materials as well as many of the polymers specifically designed for gene delivery. As a result, PEI has served as a model material for many investigations of polymeric gene delivery mechanisms. In addition, PEI and its derivatives have been used in a number of gene therapy clinical trials [3, 4]. Various explanations have been proposed for the success of PEI as a gene delivery vector. It was first proposed that, following endocytosis, PEI efficiently escapes into the cytosol due to its high buffering capacity causing osmotic swelling and rupture of acidified endocytic vesicles (the proton-sponge mechanism) [5], allowing the nucleic acids to avoid trafficking to lysosomes (LYs) where they might be degraded [6]. However, it was later shown that the acidification provided by LYs is essential for efficient delivery by PEI [7]. Our group demonstrated a lack of correlation between 
the efficiency of PEI-mediated DNA delivery and the buffering capacity of the vectors [8]. Furthermore, the endocytic pathway through which PEI/DNA vectors enter cells has been shown to control subsequent intracellular processing and strongly influences gene delivery efficiency [ 9 , 10]. Understanding the efficiency of PEI/DNA vectors will require better understanding of the cellular uptake and intracellular trafficking mechanisms.

A number of tools have been employed to study uptake and intracellular trafficking of nanoparticle drug delivery systems such as PEI/DNA polyplexes. Endocytosis pathways are often investigated by observing the effects of chemical inhibitors on cellular internalization and gene delivery efficiency. Interpretation of such experiments is difficult, however, because the available inhibitors are not necessarily specific for the targeted pathway(s) [11]. Other approaches include the expression of dominant negative variants of proteins involved in membrane trafficking [12-14] or the inhibition of the expression of such proteins by RNA interference [9]. Such studies are confounded by the interconnected nature and the complexity of endocytosis and membrane trafficking mechanisms $[15,16]$. It is desirable to study endocytosis and intracellular trafficking of gene delivery vectors with the least interference to the biology of the process.

It is also common to investigate nanoparticle uptake and intracellular trafficking by fluorescence microscopy. For example, colocalization of fluorescently labeled particles with intracellular structures labeled by immunostaining or expression of marker protein-fluorescent protein fusions is often employed to identify subcellular localization of nanoparticles. Quantitative analysis of colocalization of two fluorescently labeled entities is difficult, however. [17]. To achieve reasonable quantitative estimations, the processing of the samples, the 
calibration of the microscope, the acquisition of the images and their analysis should be done with care [18]. Unfortunately, there is no consensus on the best practices. For example, even after extensive processing of confocal micrographs to improve their resolution, some report colocalization of two labeled objects to be "significant" in the case that over $85 \%$ of their shared pixels overlap [7], while others set this value to $40 \%$ [19].

The location of particles within different organelles can also be determined after the organelles have been separated by subcellular fractionation in density-gradient centrifugation [20-22]. This method is experimentally challenging and requires careful optimization [23, 24]. Identification of organelles within the gradient after centrifugation is difficult, particularly when the objective is to separate endocytic vesicles, since their density does not vary greatly and they share many marker proteins $[25,26]$. Nevertheless, several researchers have used subcellular fractionation to investigate intracellular trafficking kinetics of gene delivery vectors [22, 27-30].

We have developed a new approach for rapidly investigating intracellular trafficking of endocytosed nanoparticles that is quantitative and less experimentally demanding than conventional methods. The method relies on polymerization of 3,3'-diaminobenzidine (DAB) by endocytosed horseradish peroxidase (HRP), causing an increase in the density of the polymerized vesicles, making vesicle resistant to detergenent and quenching fluorophores within the vesicles, all of which makes them easy to separate. We have used this method to investigate intracellular trafficking of polymer/siRNA polyplexes and compared the results to confocal microscopy and conventional subcellular fractionation.

\section{MATERIALS AND METHODS}




\section{Cells and Nucleic Acids}

A cervical cancer cell line constituitively expressing luciferase (HeLa-luc) was a kind gift from Prof. Mark E. Davis at Caltech. HeLa-luc cells were grown in DMEM with 10\% (v/v) fetal bovine serum (FBS) (Gemini Bio-Products, West Sacramento, CA) at $37^{\circ} \mathrm{C}$ and $5 \% \mathrm{CO}_{2}$ according to ATCC protocols without antibiotics or fungicides. DMEM and phosphate buffered saline (PBS) were prepared by the Cell Culture Media Facility, School of Chemical Sciences, University of Illinois. The luciferase-targeting siRNA, siGL3 (sense sequence: 5'-CUU ACG CUG AGU ACU UCG A dTdT-3'), was purchased from Dharmacon (Lafayette, CO). AllStars negative control siRNA fluorescently tagged with AlexaFluor 647 (AF647-siRNA) was purchased from Qiagen (Germantown, MD). Plasmids encoding red fluorescent protein fusions with LAMP-1 (\#181738), Rab7 (\#14436) and Rab5 (\#1443739), and wild-type Rab5 (\#13050), dominant negative Rab5 (\#13051), wild-type Rab7 (\#12661), dominant negative Rab7 (\#12662), wild-type Rab11 (\#12679), and dominant negative Rab11 (\#12680) were obtained from Addgene (Cambridge, MA) [12, 13, 31, 32]. Unless stated otherwise, all other materials were procured from Sigma-Aldrich (St. Louis, MO).

\section{Polyplex Formation and Transfection}

Branched 25-kDa PEI (Sigma, St. Louis, MO) was dissolved in purified water at $1 \mathrm{mg}$ / $\mathrm{mL}$ and kept at room temperature prior to being used as a delivery vector. To prepare polyplexes, an appropriate amount of $20 \mu \mathrm{M}$ siRNA in RNase-free water was added to PIPES buffer (20 mM PIPES, $150 \mathrm{mM} \mathrm{NaCl}, \mathrm{pH}$ 7.4) and mixed with an equal volume of PEI solution to give a final PEI:siRNA weight ratio of 10:1 (N/P 77). Polyplexes were allowed to form at room temperature 
for $20 \mathrm{~min}$ before the transfections.

HeLa-luc cells were seeded at $1 \times 10^{5}$ cells/well in 12 -well plates $24 \mathrm{~h}$ prior to transfection. Growth media was replaced with serum-free media $30 \mathrm{~min}$ prior to the addition of $50 \mu \mathrm{l}$ of the polyplex suspension to each well to achieve final concentration of $5 \mathrm{nM}$ of siRNA. The plates were incubated for $4 \mathrm{~h}$ at $37^{\circ} \mathrm{C}$ and $5 \% \mathrm{CO}_{2}$ and washed with $37^{\circ} \mathrm{C}$ PBS, and the media was replaced with fresh growth media. Twenty hours after the transfection, the cells were assayed for luciferase expression using the Promega luciferase assay system (Promega, Madison, WI) according to the manufacturer's instructions. Luciferase activity was normalized by total protein content measured using a BCA assay (Pierce, Rockford, IL). Knockdown is calculated as $1-(\mathrm{RLU} / \mathrm{mg}) /(\mathrm{RLU} / \mathrm{mg})_{\text {control, }}$, where (RLU/mg) $)_{\text {control }}$ is the luciferase activity after transfection with the control siNeg siRNA.

\section{Cellular Internalization}

In order to quantify the uptake of polyplexes, HeLa-luc cells were transfected with AF647-siRNA as described above. A highly sensitive fluorometric detection method [33] was used in combination with an efficient method [34] of removal of cationic non-internalized particles from cell membranes to determine the amount of internalized siRNA and total amount of siRNA that became associated with cells but did not internalize. Briefly, the cells were transfected for different time intervals, then half of the samples was washed with ice-cold PBS twice while the other half was washed once with an ice-cold solution of $100 \mathrm{IU} / \mathrm{ml}$ of heparin in PBS followed by a PBS wash. The cells were kept on ice during the washing steps to prevent energy-dependent internalization or recycling of particles, and each washing step lasted for 5 
min. The cells were then lysed with $200 \mu \mathrm{l}$ of lysing solution (1\% Triton X-100, 2\% SDS in PBS) and placed on an orbital shaker for $30 \mathrm{~min}$. Serial dilutions of known siRNA concentration stock were prepared in the same lysing solution in black 96-well plates, and $100 \mu 1$ of each lysate sample were added to the plates and diluted with $200 \mu \mathrm{l}$ of PBS prior to being evaluated using 620/670 nm ex/em filters on Tecan Infinite F200 Pro fluorometer (Männedorf, Switzerland).

\section{Confocal Laser Scanning Microscopy}

Endocytic vesicles were fluorescently labeled using a BacMam system (Thermo Fisher Scientific, Waltham, MA) designed to incorporate GFP-tagged Rab5, Rab7 and LAMP1 proteins into membranes of early endosomes (EE), late endosomes (LE) and lysosomes (LY), respectively. HeLa-luc cells were seeded on microscope coverslips in 6-well plates at $1 \times 10^{5}$ cells/well. On the next day, the cells were transfected for $4 \mathrm{~h}$ with the BacMam constructs according to the manufacturer's instructions. The cells were washed with PBS and allowed to incubate with fresh culture media for another $20 \mathrm{~h}$. The cells were transfected with polyplexes containing AF647-siRNA (final siRNA concentration of $5 \mathrm{nM}$ ) as described above, and at various times post-transfection (5-240 min) the cells were washed twice with ice-cold PBS, once with $0.001 \%$ SDS in PBS solution, then once with PBS, fixed with $3.7 \%$ formaldehyde in PBS solution for 5 min, washed with PBS again and mounted on microscope slides using ProLong Gold Antifade reagent (Thermo Fisher Scientific, Waltham, MA) that contained DAPI for nuclear staining. The slides were sealed with nail polish, and 10 focal planes were imaged using LSM 700 microscope (Zeiss, Oberkochen, Germany) to cover the depth of the cells. Thresholded deconvolution [35] was applied to the images, and a numerical colocalization analysis was 
performed using Coloc_2 plugin in Fiji software [36] on eight separate cells at each time point (the chosen cells were deemed to be representative of the population on each slide and based on the quality of the staining and the resulting image). To verify the procedure, Mander's colocalization coefficients (MCC) of computer-generated images with known colocalization values [37] were processed in a similar manner to the experimentally acquired images. Linear interpolation analysis was performed on the computer-generated images, and the mathematical relation of the linear section of the graph was applied toward the experimental data to obtain the corrected colocalization values.

Subcellular Fractionation by Density-Gradient Ultracentrifugation

Self-generating density gradient colloidal suspension (Percoll) was used to separate endocytic vesicles based on their sedimentation rates [38-40]. Preliminary experiments identified the position of EEs, LEs and LYs in the gradients by fractionation of lysates from cells expressing RFP fusions with Rab5, Rab7 and LAMP1, respectively (Supplementary Information). HeLa-luc cells were transfected as described above. At appropriate times posttransfection, the cells were washed with ice-cold PBS, a solution of $100 \mathrm{IU} / \mathrm{ml}$ of heparin in PBS (HS) and an additional PBS wash. The cells were mechanically scraped into $2 \mathrm{~mL}$ of homogenization media containing $0.25 \mathrm{M}$ sucrose in $10 \mathrm{mM}$ HEPES buffer $(\mathrm{pH}$ 7.6) supplemented with protease inhibitor cocktail containing AEBSF, aprotinin, bestatin, E-64, leupeptin, and pepstatin A (Sigma-Aldrich). The cells were homogenized with a Dounce homogenizer for 30 strokes, and the post-nuclear supernatant (PNS) was generated by centrifugation of homogenate at $1000 \mathrm{~g}$ for $10 \mathrm{~min}$. Twelve and a half milliliters of $20 \%$ Percoll 
solutions in homogenization solution were placed in $13.5-\mathrm{ml}$ tubes and carefully overlaid with $0.7 \mathrm{ml}$ of PNS. The tubes were sealed and centrifuged in a $70.1 \mathrm{Ti}$ Beckman rotor (Brea, CA) at $33000 \mathrm{rpm}(75,000 \mathrm{xg})$ for $30 \mathrm{~min}$ at $4^{\circ} \mathrm{C}$. The $0.3-\mathrm{ml}$ fractions were collected from the top of the tubes. To measure the total protein content and the $\beta$-hexosaminidase (lysosome marker) activity in fractions, HeLa-luc cells from four dishes were collected in $2 \mathrm{ml}$ of homogenization buffer and fractionated as described above. The $50 \mu 1$ samples from each fraction were mixed with $200 \mu \mathrm{l}$ of PBS. BCA assay was used to determine total protein content. $\beta$-hexosaminidase activity was quantified utilizing the 4-nitrophenyl-N-acetyl- $\beta$-D-glucosaminide substrate as described elsewhere [41]. The background absorbance caused by the precipitation of the Percoll particles in both of the assays was corrected by control sample that did not contain any cells.

To monitor the location of free siRNA and siRNA bound to plasma membranes, two 100mm plates of HeLa-luc cells were transfected for 15 min with PEI/AF647-siRNApolyplexes. One plate was washed with PBS three times, while the washing of the other plate included HS as described above.

To estimate the density of the collected Percoll fractions, the relationship between the index of refraction and the manually measured density provided by the manufacturer was verified. Then a linear relationship between the indices of refraction and the absorbance at 340 $\mathrm{nm}$ was established. The absorbance at $340 \mathrm{~nm}$ of each collected fraction was measured using Tecan Infinite F200 Pro instrument. The absorbance values were converted to density units by applying a linear regression from a standard curve.

\section{DAB-HRP Cytochemistry}


Polymerization of 3,3'-diaminobenzidine (DAB) by horseradish peroxidase (HRP) in the presence of $\mathrm{H}_{2} \mathrm{O}_{2}$ was used to detect intracellular location of polyplexes. HeLa-luc cells were seeded in 6-well plates at concentration of $3 \times 10^{5}$ cells/well. After $20 \mathrm{~h}$, the culture media was exchanged with $2 \mathrm{~mL}$ of serum-free media 30 min before transfection. The cells were transfected with PEI/AF647-siRNA polyplexes (5 $\mathrm{nM}$ siRNA) as described above. To investigate the fraction of delivered siRNA in EEs, the transfections were carried out for different time intervals, and HRP at final concentration of $1 \mathrm{mg} / \mathrm{ml}$ was added to cells 5 min before the end of the transfections. To label the LYs, the cells were loaded for 30 min with HRP with 90 min chase before the siRNA transfections. To label the entire endocytic system, HRP was added to cells $2 \mathrm{~h}$ before the transfection, and the transfection was carried out in the presence of HRP.

At the end of the transfection period, the cells were then washed twice with PBS and trypsinized with $500 \mu \mathrm{L}$ of dye-free trypsin solution. The following steps were performed on ice with pre-chilled solutions and microcentrifuge at $4^{\circ} \mathrm{C}$. One milliliter of PBS with $10 \%$ FBS were added to each well, and the cells were centrifuged at $500 \mathrm{~g}$ for $5 \mathrm{~min}$. The supernatant was removed, and cells were re-suspended in $2 \mathrm{~mL}$ of PBS; each sample was split into two new tubes. An additional $1 \mathrm{~mL}$ of PBS was added to each tube, and the cells were centrifuged at $700 \mathrm{~g}$ for $5 \mathrm{~min}$. The PBS was decanted, and the cells were resuspended in $0.5 \mathrm{~mL}$ of freshly prepared, $0.4-\mu \mathrm{m}$ filtered $0.3 \mathrm{mg} / \mathrm{ml} \mathrm{DAB}$ solution (in $150 \mathrm{mM} \mathrm{NaCl}, 1 \mathrm{mM} \mathrm{CaCl}, 5 \mathrm{mM} \mathrm{KCl}, 1 \mathrm{mM}$ $\mathrm{MgCl}_{2}, 20 \mathrm{mM}$ HEPES, $\mathrm{pH}$ 7.4). The cells were incubated at $4^{\circ} \mathrm{C}$ for $15 \mathrm{~min}$, and $5 \mu \mathrm{L}$ of $3 \%$ $\mathrm{H}_{2} \mathrm{O}_{2}$ was added to half of the samples. The tubes were further incubated for $30 \mathrm{~min}$ at $4^{\circ} \mathrm{C}$ with periodic gentle agitation. One milliliter of PBS was added to all of the samples, and they were centrifuged for $5 \mathrm{~min}$ at $700 \mathrm{~g}$. The liquid was decanted, and cells were washed again with $1.7 \mathrm{~mL}$ 
of PBS. The pellets were dissolved with 1\% Triton X-100 in PBS, and the tubes were vortexed and placed on an orbital shaker for $30 \mathrm{~min}$. The tubes were vortexed again, and $200 \mu \mathrm{L}$ of PBS was added. The insoluble fraction was pelleted at $17,000 \mathrm{~g}$ for $30 \mathrm{~min}$, and $200 \mu \mathrm{L}$ of the supernatant was analyzed for AF647-siRNA fluorescence as described above. The insoluble fraction was computed as a ratio of the AF647-siRNA content that was removed from the supernatant of samples to which $\mathrm{H}_{2} \mathrm{O}_{2}$ was added (sample supernatant) to the AF647-siRNA detected in matching samples in which $\mathrm{H}_{2} \mathrm{O}_{2}$ was omitted (control), as follows.

To verify the assay, cells were seeded in 6-well plates as for the transfections described above, except10-kDa dextran-AF647 conjugate (final concentration $50 \mu \mathrm{g} / \mathrm{ml}$ ) was delivered to cells instead of the siRNA. In one case, 30 min after the change of the culture media to DMEM, HRP and dextran were pre-mixed and delivered to cells simultaneously. In another case, the dextran was introduced $30 \mathrm{~min}$ after the addition of HRP. In both cases, cells were further processed as described above.

\section{RESULTS}

\section{Kinetics of PEI/siRNA uptake}

We investigated the rates of PEI/AF647-siRNA polyplex attachment to the cell membrane and cellular internalization. Cationic gene delivery vectors bind anionic proteoglycans on the cell surface $[42,43]$ and can be removed by washing the cells in the presence of heparin to compete for binding of polyplexes [34, 44, 45]. We transfected HeLa-luc cells with PEI/AF647-siRNA and compared the cell-associated fluorescence after washing in the absence and presence of 
heparin to quantify polyplexes attached to the cell surface and polyplexes internalized, respectively (Figure 1). The total siRNA associated with cells increased during the first 60 min of transfection after which it appeared to remain constant. The amount of siRNA internalized increased linearly during the entire transfection time $\left(\mathrm{R}^{2}=0.94\right)$. Approximately $20 \%$ of cellassociated siRNA was internalized during the $4 \mathrm{~h}$ transfection.

Colocalization of siRNA polyplexes with endocytic vesicle marker proteins

To identify subcellular location of internalized PEI/AF647-siRNA polyplexes, we generated cell lines expressing GFP fusions with Rab5 (indicative of EEs), Rab7 (indicative of LEs) or LAMP1 (indicative of LYs) and observed colocalization with AF647-siRNA fluorescence (Figure 2). The marker proteins could be observed in distinct punctuate structures within the cells. Qualitative observation of overlap between AF647-siRNA and GFP indicated a decrease in colocalization between Rab5 and polyplexes during the $4 \mathrm{~h}$ of transfection (Figure 2A). The amount of polyplexes colocalized with Rab7 appeared to increase up to 60 min posttransfection and decrease throughout the remainder of the transfection (Figure 2B), while colocalization with LAMP1 appeared to increase throughout the transfection (Figure 2C). 


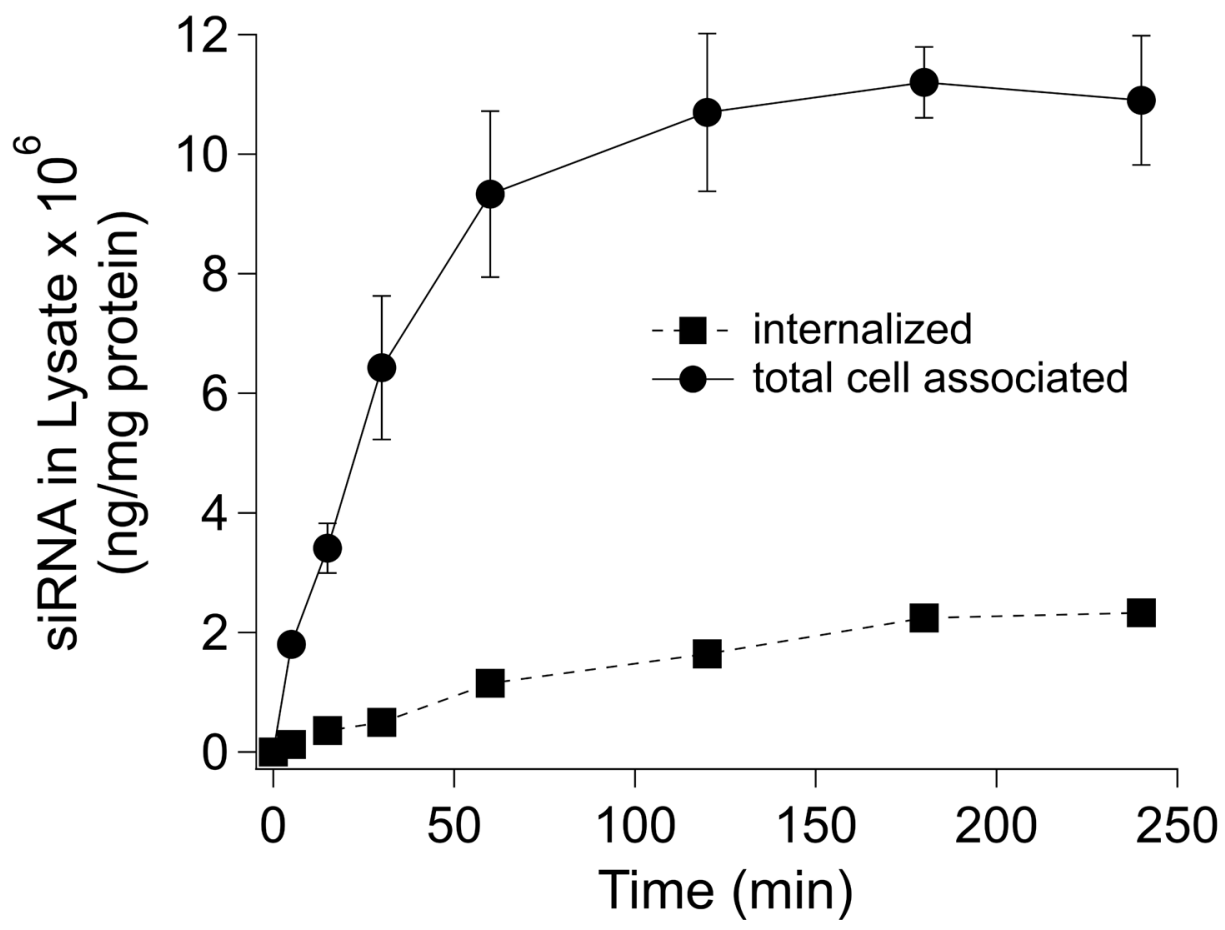

Figure 1. AF647-siRNA attachment and uptake in HeLa-luc cells at various times following transfection with PEI/AF647-siRNA polyplexes. Prior to lysis, cells were washed with PBS to quantify total cell-associated polyplexes or PBS and HS solution, removing polyplexes on the cell surface, to quantify internalized polyplexes. ( $\mathrm{N}=4$; error bars represent standard deviation.)

We quantified colocalization of polyplexes with endocytic vesicle markers by determining a Mander's coefficient for eight cells from each group (Figure 3). At the earliest time points, $\sim 70 \%$ of PEI/AF647-siRNA polyplexes colocalized with Rab5 and decreased nearly linearly $\left(\mathrm{R}^{2}=0.91\right)$ to $\sim 30 \%$ at $4 \mathrm{~h}$ post-transfection. Colocalization of AF647-siRNA with Rab7 decreased similarly, from $\sim 70 \%$ at 5 min to less than $20 \%$ at $4 \mathrm{~h}$ post-transfection. In contrast, colocalization with LAMP1 increased throughout the transfection from less than $20 \%$ to $\sim 60 \%$ during the first $30 \mathrm{~min}$ and remained at $50-60 \%$ for the remaining duration of transfection. 


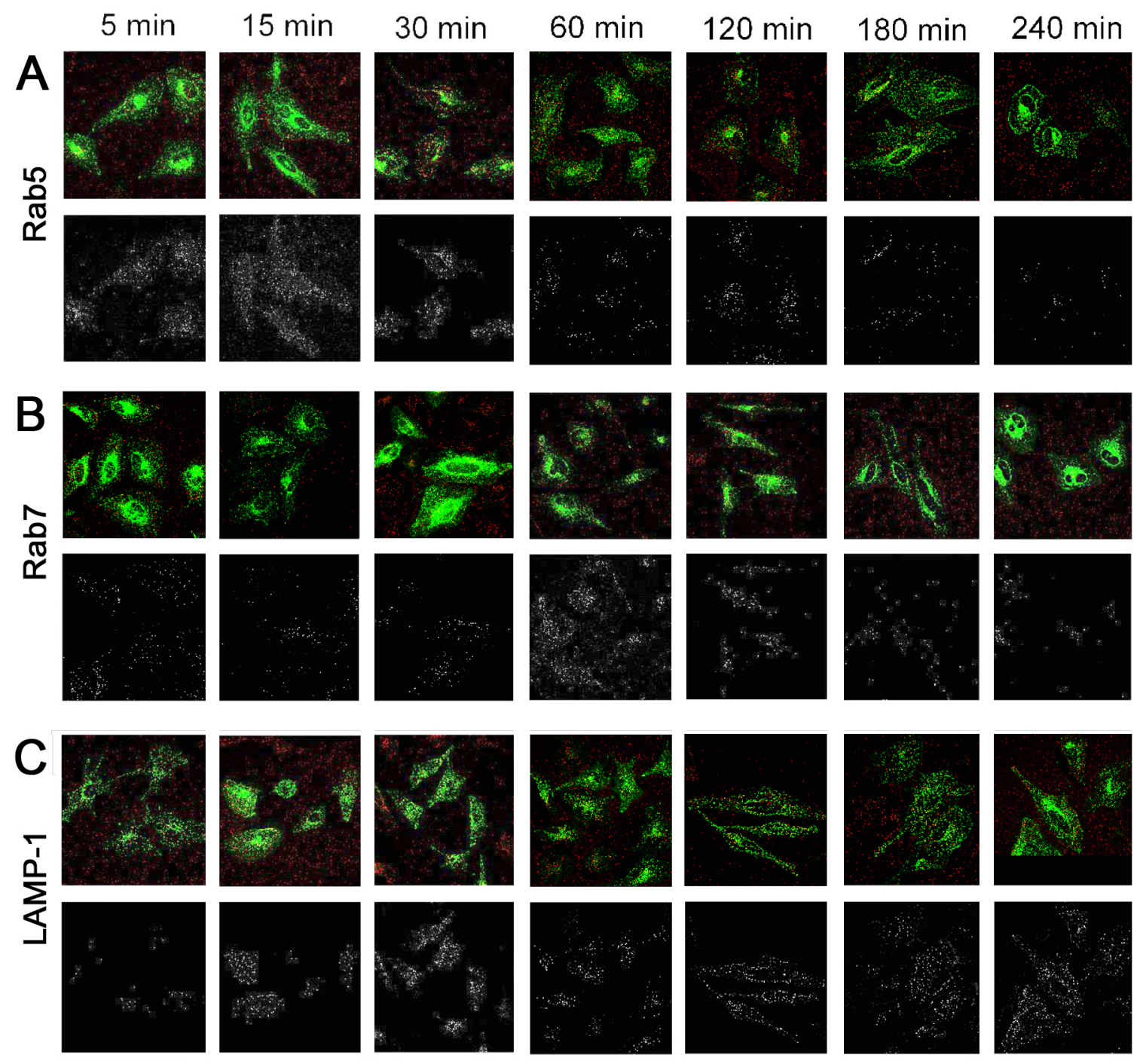

Figure 2. Confocal fluorescence micrographs of HeLa-luc cells expressing (A) GFP-Rab5, (B) GFP-Rab7 or (C) GFP-LAMP1 (green) and transfected with PEI/AF647-siRNA polyplexes (red) at various times post-transfection as indicated. Following deconvolution and addition of $10 \mathrm{z}-$ slices of the original micrographs, colocalized pixels are shown in white in the second row of each panel. All images are $203 \times 203 \mu \mathrm{m}^{2}$.

Sedimentation density-gradient fractionation of PEI/siRNA-transfected cells

Subcellular fractionation allows separation of organelles, including EEs, LEs and LYs, and can be used to identify localization of endocytosed materials in these vesicles. Ultracentrifugation of cell lysates on a $20 \%$ Percoll gradient was used to separate organelles 
based on their density and size. The locations of EEs, LEs and LYs in the gradients were determined by fractionating lysates from cells expressing RFP fusions with Rab5 (EEs), Rab7 (LEs) or LAMP1 (LYs) (Supplementary Figures 1-3). Fractions 3-10 were identified as containing EEs, fractions 11-17 contained LEs and fractions 38-42 contained LYs.
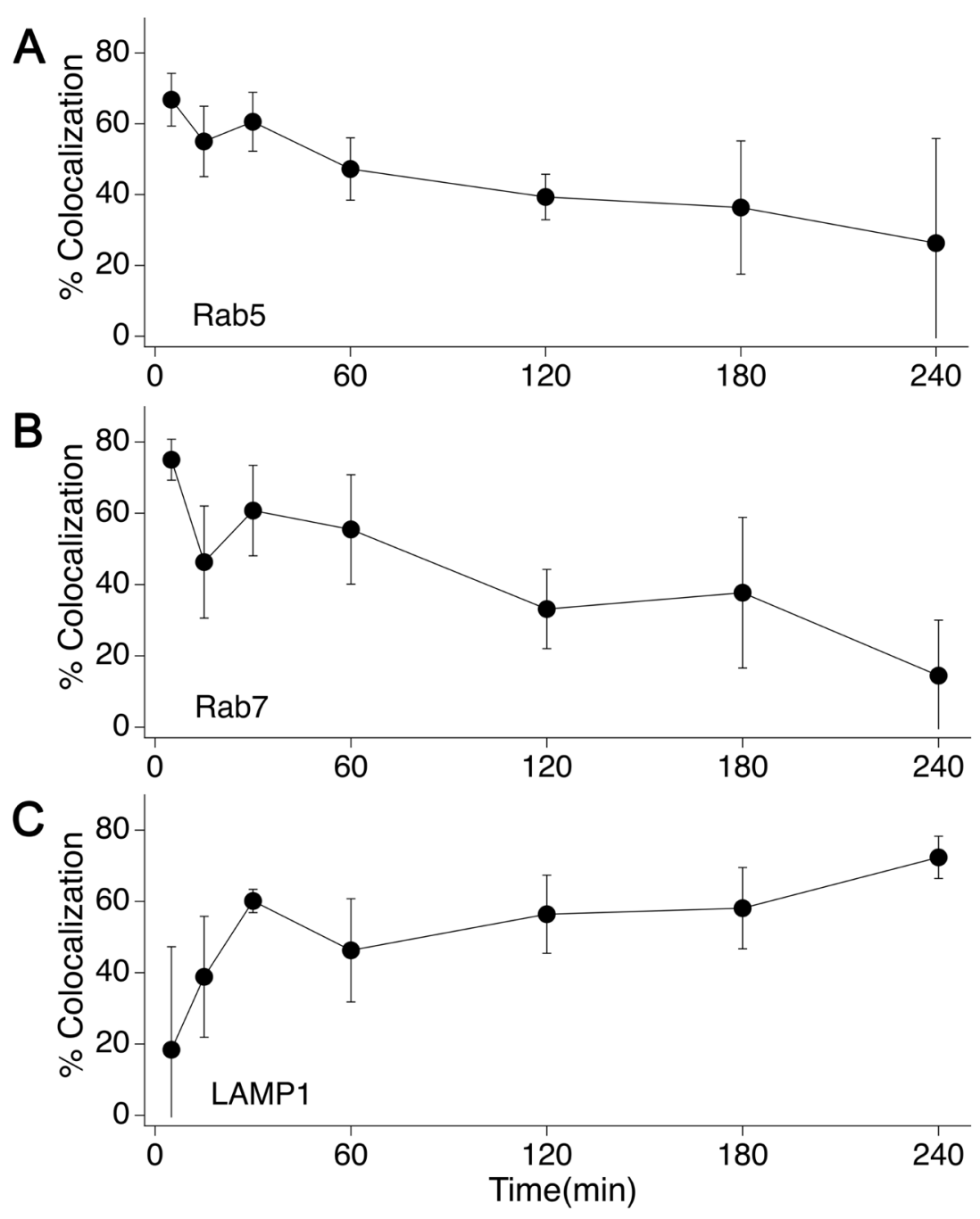

Figure 3. Colocalization (determined by Mander's coefficient) of AF647-siRNA with (A) GFPRab5, (B) GFP-Rab7 and (C) GFP-LAMP1 in HeLa-luc cells as a function of time posttransfection with PEI/AF647-siRNA polyplexes. Colocalization was determined in eight cells from three separate micrographs. (error bars represent standard deviation.)

We transfected HeLa-luc cells with PEI/AF647-siRNA polyplexes for different durations 
and determined the location of AF647-siRNA within the gradient (Figure 4A). EEs initially contained a large proportion of AF647-siRNA which decreased during the tranfsection. LEs exhibited increasing AF647-siRNA content for the first $30 \mathrm{~min}$, but the fluorescence in these fractions decreased at later times post-transfection. LYs contained little siRNA in the first 15 min of the transfection, but at $30 \mathrm{~min}$ and longer post-transfection the amount of AF647-siRNA increased in these fractions. We integrated the AF647-siRNA detected in each of these regions for each time point and normalized by the total fluorescence to determine the percentage of AF647-siRNA in each of the endocytic vesicles (Figure 4B). Approximately 45\% of AF647siRNA was found in EEs at 5 min post-transfection. The amount of AF647-siRNA in EEs decreased to $25-30 \%$ by 30 min post-transfection and remained at that level throughout the transfection. In contrast, less than 10\% of AF647-siRNA was initially located in LEs. This value increased to $\sim 20 \%$ at 15 min post-transfection then decreased to $10-15 \%$ for the remainder of the transfection. Finally, AF647-siRNA in LYs increased from $\sim 10 \%$ at $5 \mathrm{~min}$ to $\sim 25 \%$ at 60 and 120 min post-transfection.
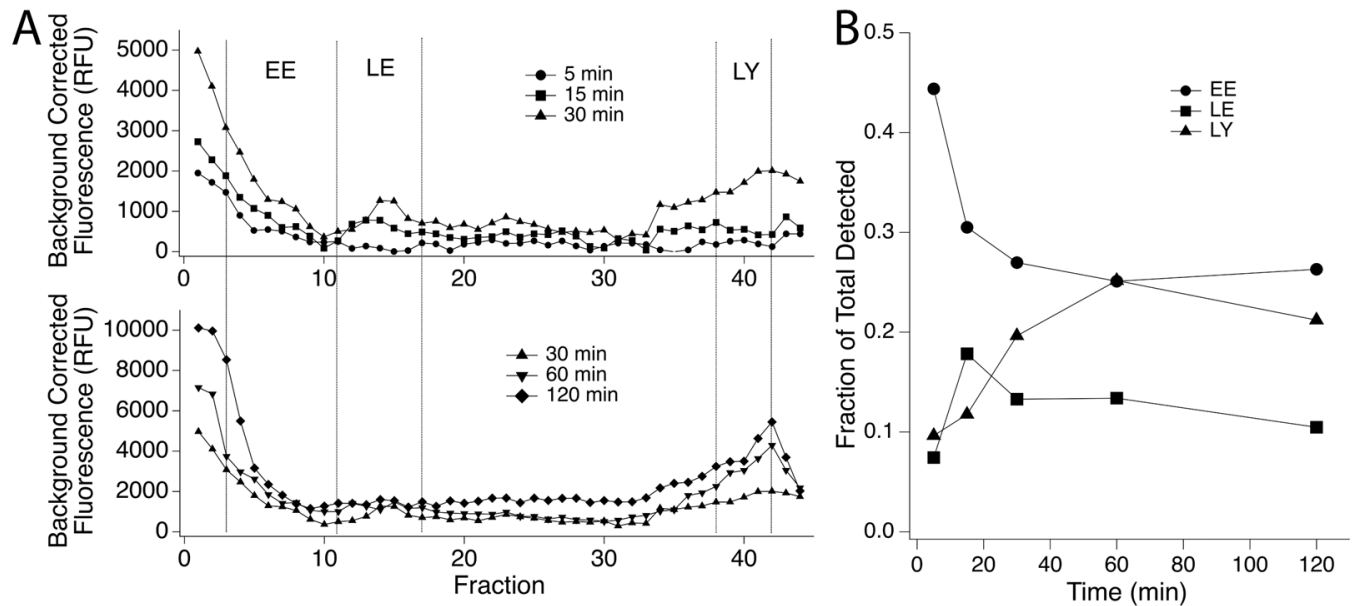

Figure 4. (A) Subcellular fractionation of HeLa-luc cells transfected with PEI/AF647-siRNA polyplexes on a $20 \%$ Percoll gradient at 5-120 min post-transfection. (B) Fraction of AF647siRNA detected in EEs (fractions 3-11), LEs (fractions 11-17) and LYs (fractions 38-42) 
determined by summing background-subtracted fluorescence in fractions corresponding to each compartment and normalizing to the summed fluorescence from all fractions. Results shown are representative of three individual experiments.

\section{DAB-HRP density-shift fractionation of PEI/siRNA-transfected cells.}

We adapted a density-shift cytochemistry method to provide quantitation of intracellular trafficking of endocytosed particles relative to the known kinetics of intracellular trafficking of HRP [46-48]. HRP is a well described marker of fluid phase pinocytosis. HRP follows the endolysosomal path upon internalization, and its activity is restricted to internal vesicles until it reaches lysosomes where it slowly degrades [49]. Membrane-permeable DAB undergoes freeradical polymerization catalyzed by HRP in the presence of $\mathrm{H}_{2} \mathrm{O}_{2}$, thus crosslinking only vesicles containing HRP. The crosslinked vesicles are relatively dense [47] and insoluble to detergent [48] and, therefore, can be easily isolated by low-speed centrifugation of detergent-lysed cells. In addition, DAB polymerization quenches fluorophores trapped within the vesicles $[50,51]$.

We transfected HeLa-luc cells with PEI/AF647-siRNA polyplexes for various durations, and the insoluble fraction was measured to determine the relative fractions of AF647-siRNA in EEs and LYs as a function of time post-transfection (Figure 5A). Approximately $60 \%$ of AF647siRNA was located in EEs 5 min post-transfection. This fraction in EEs decreased to $20 \%$ at 30 min post-transfection, remained nearly constant for the next hour, and decreased to less than $5 \%$ by $3 \mathrm{~h}$ post-transfection. AF647-siRNA was first detected in LYs 30 min after the beginning of the transfection. The presence of AF647-siRNA in LYs was relatively constant at $\sim 20 \%$ from 30 to $120 \mathrm{~min}$ and increased to nearly $40 \%$ during the last hour of the transfection. When the entire endolysosomal pathway was loaded with HRP, $60 \%$ of AF647-siRNA colocalized with HRP at 5 
min; this increased to $80 \%$ and remained constant until the end of the transfections.
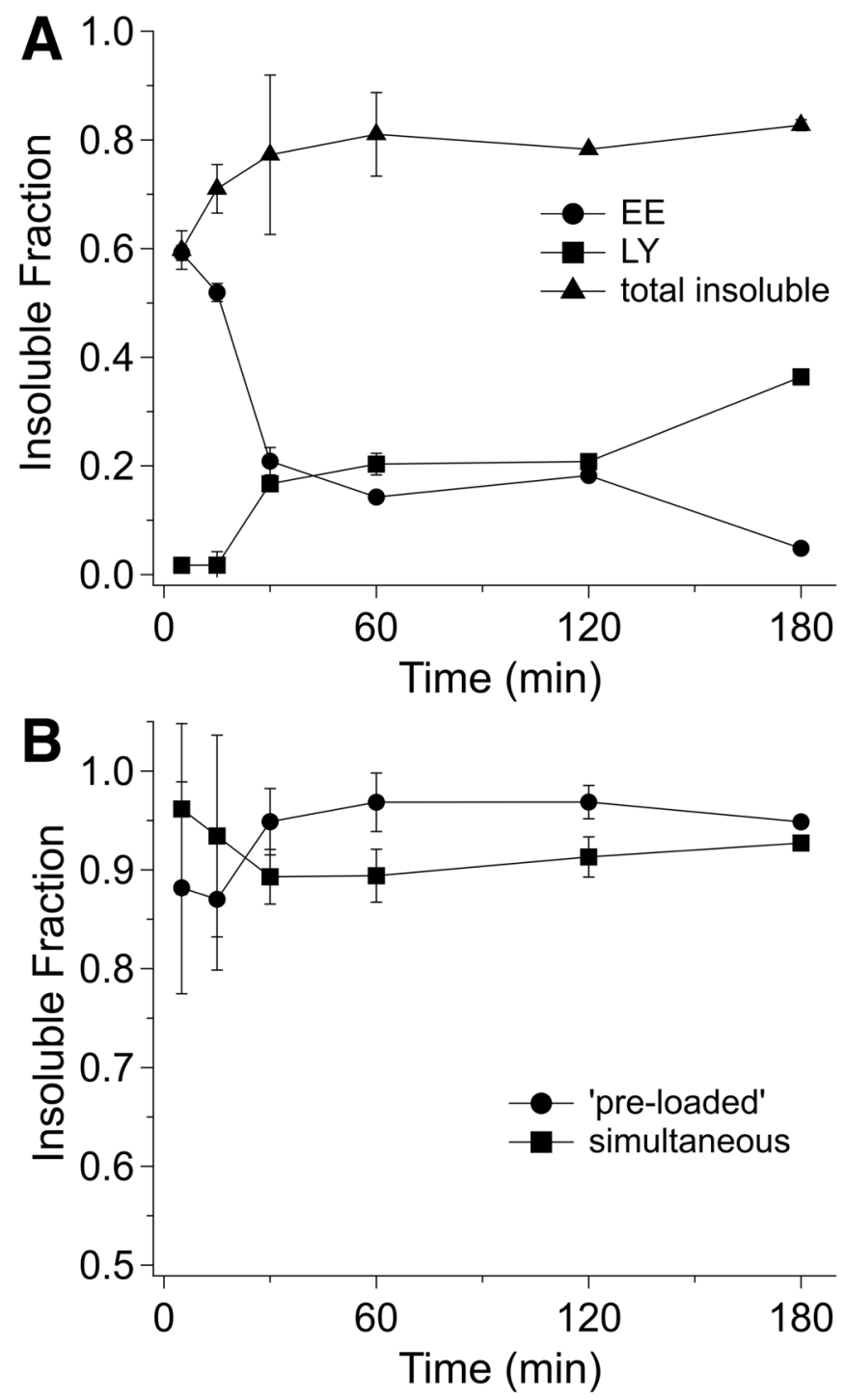

Figure 5. (A) Fraction of AF647-siRNA in early endosomes, lysosomes and all endocytic vesicles in HeLa-luc cells transfected with PEI/AF647-siRNA polyplexes measured by HRPDAB density-shift fractionation at various times post-transfection. (B) Fraction of AF647dextran colocalized with HRP introduced simultaneously or 30 min prior to dextran (pre-loaded). "Insoluble fraction" refers to the fluorescence removed with polymerized vesicles normalized by the total fluorescence in controls not given $\mathrm{H}_{2} \mathrm{O}_{2}$.

To test whether the fluorescence quenching and vesicle crosslinking was limited by the type of fluorophore or the concentration of HRP, we exposed cells to AF647-dextran conjugate 
either simultaneously with HRP or 30 min after the HRP was introduced to cells. Both dextran and HRP are commonly used markers for fluid-phase endocytosis and are not rapidly recycled. As HRP enters the cells, it first travels to the EEs using primary endocytic vesicles. The concentration of HRP in EEs then increases until the EEs stop accepting incoming cargo and mature to LEs [52]. Similar behavior is expected in the subsequent endocytic compartments. Preloading the cells with HRP for 30 min saturated the intracellular compartments with HRP before the arrival of dextran to these compartments. Since the crosslinking efficiency did not vary substantially between the two groups (Figure 5B), it is evident that the concentration of HRP within endocytic compartments does not influence the crosslinking efficiency by more than $\sim 5 \%$. Assuming that dextran does not have capacity to escape from the endolysosomal vesicles [25], the comparison between the highest insoluble fraction of dextran ( 95\%) and PEI/AF647siRNA $(\sim 80 \%)$ reveals that only up to $15 \%$ of polyplexes that entered the cells was not within internal membranes at $>30$ min post-transfection, increasing from $\sim 25 \%$ at the beginning of the transfections. This might appear counterintuitive, but the total number of internalized particles continues to increase throughout the experiment, so the number of non-vesicles-enclosed particles increases as well.

\section{DISCUSSION}

Endocytosis of particles and subsequent intracellular processing is central to the development of effective gene medicines. Much research has focused on the ability of the vectors to facilitate their release from endocytic vesicles [53]. Numerous hypotheses that attempt to describe the interactions of vector materials with internal membranes yielded limited guidelines 
for the structure of effective materials [54]. It is difficult to clearly define the link between the chemical structure and endocytic escape function of vectors without better understanding of the fate of the internalized particles. Thus a method was developed that allows simple and rapid investigation of the trafficking of polyplexes through the endolysosomal pathway.

Once attached to the cell surface, nanoparticles may be endocytosed along with their receptor proteins or may be internalized by directly inducing deformations in the cell membrane [16]. The association of PEI/siRNA polyplexes with the cell surface increased in a linear manner in the first $60 \mathrm{~min}$ of transfection but slowed significantly thereafter, presumably due to saturation of cell surface binding sites. Polyplex internalization, in contrast, increased linearly over the duration of the transfection (Figure 1). The constant rate of the internalization suggests that the cellular uptake is limited by the rate of the predominant endocytosis pathway.

Confocal fluorescence microscopy can be an effective method to investigate many biological processes including intracellular trafficking and subcellular localization of endocytosed cargo. Subcellular localization is commonly determined by imaging fluorescent cargo and marker proteins in a pseudo-colored image. The visual overlap of the cargo and markers is often taken as evidence of spatial colocalization. However, this approach often does not consider the vertical dimension of the specimen and is valid strictly "when the fluorescence of the two probes occurs in a fixed and nearly equal proportion" [19, 55]. Even if several z-layers are explored, and careful thresholding and deconvolution are performed, the resolution of most confocal microscopes is only sufficient to track large aggregates of fluorescent particles [19]. Thus, these conventional methods provide qualitative trends in colocalization of delivered particles and internal compartments such as in Figure 2. More importantly, because of the high 
density of labeled internal organelles, colocalization values may be overestimated; as the overall number of pixels of one color increases, there is an increased chance of an apparent overlap even if the two labeled structures are not spatially colocalized. Therefore, statistical analyses should be employed in conjunction with different colocalization coefficients to determine that the overlap is not occurring by chance [55].

To compensate for this issue, a correlation between the Mander's correlation coefficient (MCC) and actual colocalization was constructed (Supplementary Figure 4). The MCC, ranging from zero (no overlap) to one (complete overlap), was used because it is intuitive and convenient. Unlike other colocalization coefficients, MCC is not dependent on the relative fluorescence intensities of the investigated objects, and it has been suggested as the most appropriate quantitative colocalization parameter when dissimilar objects such as polyplexes and endosomes are spatially correlated [56]. The quantitation of colocalization from the MCC revealed that the colocalization patterns between the particles and the EEs and between the particles and LEs (Figure 3) are very similar. This could be the result of non-specific labeling of EEs and LEs by Rab5 and Rab7 as these proteins can be found in both types of vesicles, with Rab5 more prevalent in EEs and Rab7 more prevalent in LEs. Furthermore, colocalization determined from these images is only semi-quantitative due to the necessarily small sample number (eight cells for each point) and could not provide sufficiently accurate and reliable data for a robust quantitative description of intracellular trafficking kinetics. This is not an uncommon result. One study that investigated the intracellular trafficking of polyplexes in HeLa cells and included advanced techniques for spatial correction of acquired confocal 3-D images (i.e., deconvolution with experimentally determined chromatic shift corrections) resulted in only 
general trends sufficient to compare kinetics (three time points) of two polymer vectors [56].

As an alternative to microscopy, endocytic vesicles and polyplex cargo were physically separated by subcellular fractionation on a self-generating density gradient. The inherent difficulty of subcellular fractionation is a result of the requirement to physically break the cells before separating the intact organelles based on their density or sedimentation properties. The forces involved in the breaking of the plasma membrane may rupture or otherwise damage internal vesicles, which has been implicated in up to $\sim 20 \%$ variation between the results of fractionation experiments [57, 58]. In addition, endocytic vesicles continuously evolve, making them difficult to define based on their physical or chemical properties. siRNA located in the cytosol would be expected to be located in the topmost fractions within the gradients. However, these fractions may have been "contaminated" with siRNA released as a result of the cell homogenization procedure as evidenced by the high $\beta$-hexosaminidase activity (Supplementary Figure 2) and high levels of RFP-Rab5, -Rab7 and -LAMP1 (Supplementary Figure 1). The origin of the siRNA in these top fractions could not be elucidated with the employed fractionation protocol.

Although the separation resolution was limited, some quantitative kinetics data could be extracted from the subcellular fractionation (Figure 4B). The amount of siRNA in the heaviest fractions (35-45) and the lightest fractions (0-10) increased throughout the experiment . However, the fraction of siRNA located within each compartment was nearly constant after 30-60 min post-transfection. Still, the data only describe $\sim 60 \%$ of the detected siRNA, and the identified representative regions within the gradients might include other organelles that participate in intracellular trafficking (e.g., recycling endosomes, autophagosomes, hybrid 
vesicles, Golgi, ER, etc.). Compared to microscopy, the advantages of fractionation are in the large number of cells that are represented in each experiment and in a higher certainty that the measured fluorescence comes not only from aggregates but from all of the delivered siRNA.

Because of the limitations of fluorescence microscopy and subcellular fractionation, the DAB-HRP density shift method was developed to provide increased resolution of endocytic vesicles in density gradient centrifugation [47]. We adapted this method along with the known intracellular trafficking of HRP to determine the kinetics of polyplex accumulation in EEs and LYs (Figure 5A). The resulting data were similar to those obtained with subcellular fractionation. For example, the fraction of siRNA in EEs decreased rapidly during the first $30 \mathrm{~min}$ and remained relatively constant thereafter. The $60 \%$ of polyplexes localized in EEs at the first measured point $(5 \mathrm{~min}$ ) should be considered in the context of the maximum possible siRNA that could be removed when the total endolysosomal system was loaded with HRP, which was also $60 \%$. This may be the result of polyplexes and HRP being internalized via different mechanisms such that they are initially present in different vesicles but merge in EEs after 5-10 min [59]. This can be reinforced by the fact that over $85 \%$ of the delivered dextran was detected in the vesicles that also contained HRP 5 min after both were simultaneously introduced to cells (Figure 5B). Both dextran and HRP are similar types of pinocytic endocytosis markers that readily enter cells through multiple pathways [60-62]. Nanoparticles formed of cationic polymers, however, are more likely to enter cells through a more specific pathway [63].

The presence of siRNA in LYs increased during the first $30 \mathrm{~min}$ of transfection and stayed constant at $\sim 20 \%$ during the subsequent $90 \mathrm{~min}$ (Figure $5 \mathrm{~A}$ ). This result is similar to the trafficking of polyplexes into LYs observed with density-gradient fractionation (Figure 4B). It is 
not reasonable to expect $10 \%$ of delivered particles to reach LYs only 5 min after the delivery as was observed with fractionation, however, suggesting that the DAB-HRP cytochemistry method may be more accurate. The polyplex trafficking up to $3 \mathrm{~h}$ post-transfection was investigated only with DAB-HRP cytochemistry, which revealed an increase in the fraction of particles in LYs and a decrease in EEs. This could be explained by polyplexes following multiple intracellular pathways on their way to LYs or by the existence of hybrid LE/LY compartment that steadily accepted particles during the second hour of transfections from LEs and began transferring the cargo to LYs during the third hour. Besides the well described endolysosomal maturation, the particles could be delivered to LYs via autophagy from cytoplasm after escaping from endosomes or after entering the cells through a non-endocytic pathway [64]. This process could be slower than the typical endolysosomal processing and began providing delivered material to LYs $2 \mathrm{~h}$ after the delivery. Alternatively, since a 30 min pulse of HRP was used to mark the LYs, it is possible that not all HRP reached LYs at various times when the crosslinking reaction was initiated. It is well known that HRP accumulates in LYs [65], but if the transition between the hybrid LE/LY vesicles to terminal LYs is slow, then the DAB polymerization could trap delivered siRNA in both the pre-lysosomal and lysosomal compartments. The constant fraction of dextran detected over the course of the experiment (Figure 5B) indicated that the detected increase in amount of siRNA in LYs between 2 and $3 \mathrm{~h}$ was not the result of insufficient concentration of HRP in LYs. If the HRP concentration was only high enough to allow the detection of siRNA at that compartment $2-3 \mathrm{~h}$ from the beginning of the transfections, then a sharper jump in the "insoluble fraction" during the same period in the samples with the fully HRP-loaded endolysosomal system would have been observed (Figure 5A). In fact, since similar 
crosslinking efficiency was observed when AF647-labeled dextran was delivered to cells either simultaneously or 30 min after the HRP was introduced, the concentration of HRP within the

endocytic compartments did not substantially affect the outcomes (Figure 5B). The great sensitivity of the DAB cytometry was demonstrated by a very elegant study by Stinchcombe et al., which showed that a single HRP molecule entrapped in 60-80 $\mathrm{nm}$ liposomal vesicles could be sufficient to fill the entire lumen with DAB polymerization reaction product [66].

\section{CONCLUSIONS}

Design of materials for delivery of nucleic acids will be aided by enhanced quantitative understanding of vector delivery mechanisms including endocytosis and subsequent trafficking of endocytic vesicles. Conventional methods for analysis of intracellular trafficking include fluorescence microscopy and subcellular fractionation on a density gradient. Using confocal fluorescence microscopy, we observed colocalization of polyplexes with Rab5, Rab7 and LAMP-1, markers of EEs, LEs and LYs, respectively. Quantitation of those images revealed transport of polyplexes from EEs to LYs over the course of $4 \mathrm{~h}$ post-transfection. The sample size that can be investigated is small, however, and complex image analysis is required to obtain quantitative data. We observed similar kinetics of polyplex transport through the endocytic pathway by subcellular fractionation. But this method is greatly limited by the experimental difficulties in preparing the density gradients and isolating intact organelles, by the long duration of high speed centrifugation needed to separated endocytic vesicles, and by the limited resolution of the separation. Thus, we described a method for quantifying endocytic trafficking of polyplexes based on DAB polymerization by HRP within endocytic vesicles. The polymerized 
vesicles are stable to detergent and dense, making them easy to isolate by complete cellular lysis and low speed centrifugation. As a result, a large number of samples can be processed efficiently and quickly. This method demonstrated kinetics of endocytic vesicle transport of polyplexes that was consistent with the conventional methods. We expect this method will be valuable for understanding mechanisms of polyplex-mediated nucleic acid delivery, as well as investigation of internalization and endocytic processing of nanoparticle delivery agents more generally.

\section{ACKNOWLEDGMENTS}

This work was partially supported by the American Heart Association Predoctoral Fellowship (11PRE5960002) (ML), National Science Foundation (BES 06-02636) (DWP) and National Institutes of Health GM085222 (DWP). In addition, we thank Sandy Mattick at the Cell Culture Media Facility at the University of Illinois for help with preparation of cell media. Confocal fluorescence microscopy was performed at the Institute of Genomic Biology Core Facilities, University of Illinois, with technical help from Mayandi Sivaguru. 


\section{REFERENCES}

[1] O. Boussif, F. Lezoualch, M.A. Zanta, M.D. Mergny, D. Scherman, B. Demeneix, J.P. Behr, A versatile vector for gene and oligonucleotide transfer into cells in culture and in vivo: polyethylenimine, Proc.Natl.Acad.Sci., USA, 92 (1995) 7297-7301.

[2] B. Abdallah, A. Hassan, C. Benoist, D. Goula, J.P. Behr, B.A. Demeneix, A powerful nonviral vector for in vivo gene transfer into the adult mammalian brain: polyethylenimine, Hum. Gene Ther., 7 (1996) 1947-1954.

[3] M. Gunther, J. Lipka, A. Malek, D. Gutsch, W. Kreyling, A. Aigner, Polyethylenimines for RNAi-mediated gene targeting in vivo and siRNA delivery to the lung, Eur. J. Pharm. Biopharm., 77 (2011) 438-449.

[4] J. Zhou, K.-T. Shum, J.C. Burnett, J.J. Rossi, Nanoparticle-based delivery of RNAi therapeutics: progress and challenges., Pharmacueticals, 6 (2013) 85-107.

[5] J.P. Behr, The proton sponge: a trick to enter cells the viruses did not exploit, Chimia, 51 (1997) 34-36.

[6] W.T. Godbey, M.A. Barry, P. Saggau, K.K. Wu, A.G. Mikos, Poly(ethylenimine)-mediated transfection: a new paradigm for gene delivery, J. Biomed. Matls. Res., 51 (2000) 321-328.

[7] A.T. Dinh, C. Pangarkar, T. Theofanous, S. Mitragotri, Understanding intracellular transport processes pertinent to synthetic gene delivery via stochastic simulations and sensitivity analyses, Biophys. J., 92 (2007) 831-846.

[8] M.L. Forrest, D.W. Pack, On the kinetics of polyplex endocytic trafficking: implications for gene delivery vector design, Mol. Ther., 6 (2002) 57-66.

[9] N.P. Gabrielson, D.W. Pack, Efficient polyethylenimine-mediated gene delivery proceeds via a caveolar pathway in HeLa cells, J. Controlled Rel., 136 (2009) 54-61.

[10] J. Rejman, A. Bragonzi, M. Conese, Role of clathrin- and caveolae-mediated endocytosis in gene transfer mediated by lipo- and polyplexes, Mol. Ther., 12 (2005) 468.

[11] D. Vercauteren, R. Vandenbroucke, A. Jones, J. Rejman, J. Demeester, S. De Smedt, The Use of Inhibitors to Study Endocytic Pathways of Gene Carriers: Optimization and Pitfalls, Mol. Ther., 18 (2010) 561-569.

[12] D.K. Sharma, A. Choudhury, R.D. Singh, C.L. Wheatley, D.L. Marks, R.E. Pagano, Glycosphingolipids internalized via caveolar-related endocytosis rapidly merge with the clathrin pathway in early endosomes and form microdomains for recycling, J. Biol. Chem., 278 (2003) 7564-7572.

[13] A. Choudhury, M. Dominguez, V. Puri, D.K. Sharma, K. Narita, C.L. Wheatley, D.L. Marks, R.E. Pagano, Rab proteins mediate Golgi transport of caveola-internalized glycosphingolipids and correct lipid trafficking in Niemann-Pick C cells, J. Clin. Invest., 109 (2002) 1541-1550. 
[14] C. Hopkins, A. Gibson, J. Stinchcombe, C. Futter, Chimeric molecules employing horseradish peroxidase as reporter enzyme for protein localization in the electron microscope, in: J. Thorner, S.D. Emr, J.N. Abelson (Eds.) Applications of Chimeric Genes and Hybrid Proteins Pt B: Cell Biology and Physiology, 2000, pp. 35-45.

[15] J. Huotari, A. Helenius, Endosome maturation, EMBO J., 30 (2011) 3481-3500.

[16] A. Akinc, G. Battaglia, Exploiting Endocytosis for Nanomedicines, Cold Spring Harbor Perspectives in Biology, 5 (2013).

[17] S.N. Xiang, H.J. Tong, Q. Shi, J.C. Fernandes, T. Jin, K.R. Dai, X.L. Zhang, Uptake mechanisms of non-viral gene delivery, J. Controlled Rel., 158 (2012) 371-378.

[18] J. Adler, I. Parmryd, Colocalization Analysis in Fluorescence Microscopy, in: J.D. Taatjes, J. Roth (Eds.) Cell Imaging Techniques: Methods and Protocols, Humana Press, Totowa, NJ, 2013, pp. 97-109.

[19] J. Gilleron, W. Querbes, A. Zeigerer, A. Borodovsky, G. Marsico, U. Schubert, K. Manygoats, S. Seifert, C. Andree, M. Stoter, H. Epstein-Barash, L.G. Zhang, V. Koteliansky, K. Fitzgerald, E. Fava, M. Bickle, Y. Kalaidzidis, A. Akinc, M. Maier, M. Zerial, Image-based analysis of lipid nanoparticle-mediated siRNA delivery, intracellular trafficking and endosomal escape, Nature Biotechnology, 31 (2013) 638-U102.

[20] L.R. Walker, H.A.M. Hussein, S.M. Akula, Subcellular fractionation method to study endosomal trafficking of Kaposi's sarcoma-associated herpesvirus, Cell Biosci., 6 (2016) 10.

[21] J.M. Graham, D. Rickwood, Subcellular Fractionation: A Practical Approach, Oxford University Press, Oxford, England, 1997.

[22] J.Y. Zhou, J.W. Yockman, S.W. Kim, S.E. Kern, Intracellular kinetics of non-viral gene delivery using polyethylenimine carriers, Pharm. Res., 24 (2007) 1079-1087.

[23] F.P. Seib, A.T. Jones, R. Duncan, Establishment of subcellular fractionation techniques to monitor the intracellular fate of polymer therapeutics I. Differential centrifugation fractionation B16F10 cells and use to study the intracellular fate of HPMA copolymer-doxorubicin, J. Drug Targeting, 14 (2006) 375-390.

[24] D. Vercauteren, J. Rejman, T.F. Martens, J. Demeester, S.C. De Smedt, K. Braeckmans, On the cellular processing of non-viral nanomedicines for nucleic acid delivery: Mechanisms and methods, J. Controlled Rel., 161 (2012) 566-581.

[25] W.H. Humphries, C.J. Szymanski, C.K. Payne, Endo-lysosomal vesicles positive for Rab7 and LAMP1 are terminal vesicles for the transport of dextran., PLOS ONE, 6 (2011) e26626.

[26] C.S. Pillay, E. Elliott, C. Dennison, Endolysosomal proteolysis and its regulation, Biochem. J., 363 (2002) 417-429. 
[27] M. Manunta, L. Izzo, R. Duncan, A.T. Jones, Establishment of subcellular fractionation techniques to monitor the intracellular fate of polymer therapeutics II. Identification of endosomal and lysosomal compartments in HepG2 cells combining single-step subcellular fractionation with fluorescent imaging, J. Drug Targeting, 15 (2007) 37-50.

[28] J. Shi, B. Chou, J.L. Choi, A.L. Ta, S.H. Pun, Investigation of Polyethylenimine/DNA Polyplex Transfection to Cultured Cells Using Radiolabeling and Subcellular Fractionation Methods, Mol. Pharm., 10 (2013) 2145-2156.

[29] M. Colin, M. Maurice, G. Trugnan, M. Kornprobst, R.P. Harbottle, A. Knight, R.G. Cooper, A.D. Miller, J. Capeau, C. Coutelle, M.C. Brahimi-Horn, Cell delivery, intracellular trafficking and expression of an integrin-mediated gene transfer vector in tracheal epithelial cells, Gene Ther., 7 (2000) 139.

[30] G.A. Banks, R.J. Roselli, R. Chen, T.D. Giorgio, A model for the analysis of nonviral gene therapy, Gene Ther., 10 (2003) 1766-1775.

[31] N.M. Sherer, M.J. Lehmann, L.F. Jimenez-Soto, A. Ingmundson, S.M. Horner, G. Cicchetti, P.G. Allen, M. Pypaert, J.M. Cunningham, W. Mothes, Visualization of retroviral replication in living cells reveals budding into multivesicular bodies, Traffic, 4 (2003) 785-801.

[32] A. Vonderheit, A. Helenius, Rab7 associates with early endosomes to mediate sorting and transport of semliki forest virus to late endosomes, PLOS Biol., 3 (2005) 1225-1238.

[33] P. Vader, L.J. van der Aa, J.F.J. Engbersen, G. Storm, R.M. Schiffelers, A method for quantifying cellular uptake of fluorescently labeled siRNA, J. Controlled Rel., 148 (2010) 106-109.

[34] M.O. Durymanov, E.A. Beletkaia, A.V. Ulasov, Y.V. Khramtsov, G.A. Trusov, N.S. Rodichenko, T.A. Slastnikova, T.V. Vinogradova, N.Y. Uspenskaya, E.P. Kopantsev, A.A. Rosenkranz, E.D. Sverdlov, A.S. Sobolev, Subcellular trafficking and transfection efficacy of polyethylenimine-polyethylene glycol polyplex nanoparticles with a ligand to melanocortin receptor-1, J. Controlled Rel., 163 (2012) 211-219.

[35] C. Vonesch, M. Unser, A fast thresholded Landweber algorithm for wavelet-regularized multidimensional deconvolution, Ieee Transactions on Image Processing, 17 (2008) 539-549.

[36] J. Schindelin, I. Arganda-Carreras, E. Frise, V. Kaynig, M. Longair, T. Pietzsch, S. Preibisch, C. Rueden, S. Saalfeld, B. Schmid, J.-Y. Tinevez, D.J. White, V. Hartenstein, K. Eliceiri, P. Tomancak, A. Cardona, Fiji: an open-source platform for biological-image analysis, Nat. Methods, 9 (2012) 676-682.

[37] V. Zinchuk, Y. Wu, O. Grossenbacher-Zinchuk, Bridging the gap between qualitative and quantitative colocalization results in fluorescence microscopy studies, Sci. Rep., 3 (2013). 
[38] T.E. Tjelle, A. Brech, L.K. Juvet, G. Griffiths, T. Berg, Isolation and characterization of early endosomes, late endosomes and terminal lysosomes: Their role in protein degradation, J. Cell. Sci., 109 (1996) 2905-2914.

[39] D.M. Underhill, D.A. Owensby, P.A. Morton, A.L. Schwartz, Endocytosis and lysosomal delivery of tissue plasminogen activator-inhibitor-1 complexes in HepG2 cells, Blood, 80 (1992) 2746-2754.

[40] W. Stoorvogel, H.J. Geuze, G.J. Strous, Sorting of endocytosed transferrin and asialoglycoprotein occurs immediately after internalization in HepG2 cells, J. Cell Biol., 104 (1987) 1261-1268.

[41] B.E. Schaub, P. Nair, J. Rohrer, Analysis of Protein Transport to Lysosomes, in: Current Protocols in Cell Biology, John Wiley \& Sons, Inc., 2001.

[42] Z.U. Rehman, I.S. Zuhorn, D. Hoekstra, How cationic lipids transfer nucleic acids into cells and across cellular membranes: Recent advances, J. Controlled Rel., 166 (2013) 46-56.

[43] G.T. Hess, W.H. Humphries, N.C. Fay, C.K. Payne, Cellular binding, motion, and internalization of synthetic gene delivery polymers, Biochem. Biophys. Acta, 1773 (2007) 1583-1588.

[44] Y. Hayashi, Y. Noguchi, H. Harashima, Non-linear pharmacokinetics of octaargininemodified lipid nanoparticles: Barriers from in vitro to in vivo, J. Controlled Rel., 161 (2012) 757-762.

[45] S. Pinel, E. Aman, F. Erblang, J. Dietrich, B. Frisch, J. Sirman, A. Kichler, A.P. Sibler, M. Dontenwill, F. Schaffner, G. Zuber, Quantitative measurement of delivery and gene silencing activities of siRNA polyplexes containing pyridylthiourea-grafted polyethylenimines, $\mathrm{J}$. Controlled Rel., 182 (2014) 1-12.

[46] P.J. Courtoy, J. Quintart, P. Baudhuin, Shift in the equilibrium density of sub-cellular organelles containing peroxidase using the diaminobenzidine procedure, J. Cell Biol., 95 (1982) A424-A424.

[47] P.J. Courtoy, J. Quintart, P. Baudhuin, Shift of equilibrium density induced by 3,3'diaminobenzidine cyto-chemistry - a new procedure for the analysis and purification of peroxidase-containing organelles, J. Cell Biol., 98 (1984) 870-876.

[48] R.S. Ajioka, J. Kaplan, Characterization of endocytic compartments using the horseradishperoxidase diaminobenzidine density shift technique, J. Cell Biol., 104 (1987) 77-85.

[49] W. Stoorvogel, Analysis of the endocytic system by using horseradish peroxidase, Trends Cell Biol., 8 (1998) 503-505.

[50] A. Zeigerer, M.A. Lampson, O. Karylowski, D.D. Sabatini, M. Adesnik, M.D. Ren, T.E. McGraw, GLUT4 retention in adipocytes requires two intracellular insulin-regulated transport steps, Mol. Biol. Cell, 13 (2002) 2421-2435. 
[51] M.A. Lampson, J. Schmoranzer, A. Zeigerer, S.M. Simon, T.E. McGraw, Insulin-regulated release from the endosomal recycling compartment is regulated by budding of specialized vesicles, Mol. Biol. Cell, 12 (2001) 3489-3501.

[52] L. Thilo, E. Stroud, T. Haylett, Maturation of early endosomes and vesicular traffic to lysosomes in relation to membrane recycling, J. Cell. Sci., 108 (1995) 1791-1803.

[53] Y.J. Kwon, Before and after Endosomal Escape: Roles of Stimuli-Converting siRNA/ Polymer Interactions in Determining Gene Silencing Efficiency, Acc. Chem. Res., 45 (2012) 1077-1088.

[54] J. Nguyen, F.C. Szoka, Nucleic Acid Delivery: The Missing Pieces of the Puzzle?, Acc. Chem. Res., 45 (2012) 1153-1162.

[55] K.W. Dunn, M.M. Kamocka, J.H. McDonald, A practical guide to evaluating colocalization in biological microscopy, Am. J. Physiol., 300 (2011) C723-C742.

[56] N.K. Reitan, B. Sporsheim, A. Bjorkoy, S. Strand, C.d.l. Davies, Quantitative 3-D colocalization analysis as a tool to study the intracellular trafficking and dissociation of pDNAchitosan polyplexes, J. Biomed. Optics, 17 (2012).

[57] D.M. Ward, R. Ajioka, J. Kaplan, Cohort movement of different ligands and receptors in the intracellular endocytic pathway of alveolar macrophages, J. Biol. Chem., 264 (1989) 8164-8170.

[58] D.M. Ward, D.P. Hackenyos, J. Kaplan, Fusion of sequentially internalized vesicles in alveolar macrophages, J. Cell Biol., 110 (1990) 1013-1022.

[59] I. Canton, G. Battaglia, Endocytosis at the nanoscale, Chem. Soc. Rev., 41 (2012) 2718-2739.

[60] M. Marsh, S. Schmid, H. Kern, E. Harms, P. Male, I. Mellman, A. Helenius, Rapid analytical and preparative isolation of functional endosomes by free-flow electrophoresis, J. Cell Biol., 104 (1987) 875-886.

[61] M.J. Buckmaster, D.L. Braico, A.L. Ferris, B. Storrie, retention of pinocytized solute by cho cell lysosomes correlates with molecular-weight, Cell. Biol. Intl. Rep., 11 (1987) 501-507.

[62] B. Shi, M. Abrams, Technologies for Investigating the Physiological Barriers to Efficient Lipid Nanoparticle-siRNA Delivery, J. Histochem. Cytochem., 61 (2013) 407-420.

[63] A. El-Sayed, H. Harashima, Endocytosis of Gene Delivery Vectors: From Clathrindependent to Lipid Raft-mediated Endocytosis, Mol. Ther., 21 (2013) 1118-1130.

[64] K. Remaut, V. Oorschot, K. Braeckmans, J. Klumperman, S.C. De Smedt, Lysosomal capturing of cytoplasmic injected nanoparticles by autophagy: An additional barrier to non viral gene delivery, J. Controlled Rel., 195 (2014) 29-36. 
[65] R.M. Steinman, J.M. Silver, Z.A. Cohn, Pinocytosis in fibroblasts - quantitative studies in vitro, J. Cell Biol., 63 (1974) 949-969.

[66] J.C. Stinchcombe, H. Nomoto, D.F. Cutler, C.R. Hopkins, Anterograde and retrograde traffic between the rough endoplasmic-reticulum and the golgi-complex, J. Cell Biol., 131 (1995) $1387-1401$. 